\title{
A Support Price Theorem for the Continuous Time Model of Capital Accumulation
} Author(s): Shin-Ichi Takekuma

Source: Econometrica, Vol. 50, No. 2 (Mar., 1982), pp. 427-442

Published by: The Econometric Society

Stable URL: http://www.jstor.org/stable/1912636

Accessed: 18-10-2017 22:02 UTC

JSTOR is a not-for-profit service that helps scholars, researchers, and students discover, use, and build upon a wide range of content in a trusted digital archive. We use information technology and tools to increase productivity and facilitate new forms of scholarship. For more information about JSTOR, please contact support@jstor.org.

Your use of the JSTOR archive indicates your acceptance of the Terms \& Conditions of Use, available at http://about.jstor.org/terms 


\title{
A SUPPORT PRICE THEOREM FOR THE CONTINUOUS TIME MODEL OF CAPITAL ACCUMULATION ${ }^{1}$
}

\begin{abstract}
SHIN-ICHI TAKEKUMA ${ }^{2}$
We consider a model of capital accumulation and prove the existence of a support price path for the optimal path of capital accumulation. The considered model is a continuous time model of infinite horizon. Our problem is the so-called convex problem of optimal control without differentiability. We adopt the overtaking optimality criterion and prove the existence of a dual price path which supports the value function as well as the Hamiltonian function.
\end{abstract}

\section{INTRODUCTION}

IN THIS PAPER we consider a model of capital accumulation and prove the existence of a support price path for the optimal path of capital accumulation. The considered model is a continuous time model of infinite horizon.

Under some assumptions of differentiability, we can obtain a dual path for the optimal path by the Euler equation, or by the maximum principle of Pontryagin [13]. (See, for example, Halkin [7] and Haurie [8].) The differentiability assumption is, however, too restrictive and excludes a large class of economic models, such as models with fixed-coefficient production technology like Leontief or von Neuman models. So, we shall not assume the differentiability, but instead we shall assume the convexity of the model, which can be justified in many economic models.

Optimal control without a differentiability assumption has been studied by Rockafellar [14] and Halkin [6] in convex models. Some mathematical generalizations in non-convex models have been done more recently by Warga [19], Aubin and Clarke [1], and so on. However, if we confine ourselves to the case of convex models, the models and results in this paper are more general than theirs. The distinct features in our results are as follows: First, our optimality criterion is a general one, that is, the so-called overtaking criterion originally introduced by von Weizsacker [18] and Gale [5]. Second, we shall prove the existence of a dual price path which supports the value function as well as the Hamiltonian function. The existence of such a price path is non-trivial, since we can easily show an example of a price path which supports the Hamiltonian function, but does not support the value function. Benveniste and Scheinkman [2] have obtained the same result in a differentiable model with a somewhat stronger "interiority" assumption on the optimal path. The fact that a price path supports both the

\footnotetext{
'This paper is based on the author's paper [15] and the author's Ph.D. dissertation [16] submitted to the University of Rochester, under supervision of Professors L. M. Benveniste and L. W. McKenzie.

${ }^{2}$ The author is grateful to Professors L. W. McKenzie and L. M. Benveniste for their suggestions and encouragement. Also, the author would like to thank Professors J.-P. Aubin, W. A. Brock, E. Sheshinski, and an anonymous referee for their comments.
} 
value function and the Hamiltonian function can be used in proving the "turnpike" property of the optimal paths (see McKenzie [10]) and in proving the transversality condition (see Takekuma [16]).

The main result in this paper is the Main Theorem in Section 4, in which the existence of a dual price path for the optimal path is proved. The theorem is a counterpart of the support price lemma proved by McKenzie [10, Lemma 1] in a discrete time model. One of the key lemmas in our argument is Lemma 6.2, which exactly corresponds to the "induction" argument by Weitzman [20] and McKenzie [9 and 10]. Of course, since our model is in continuous time, their induction procedure cannot be applied directly in our case. However, even in the case of continuous time models, their method is quite useful and actually makes the proof simpler and more elementary. A similar proof can be found in Halkin [6], but his method seems effective only for finite horizon models.

\section{MATHEMATICAL NOTATION}

The set of all positive integers is denoted by $N$. Let $R$ be the real line. $R^{n}$ denotes the $n$-dimensional Euclidean space, where $n \in N$. For any $x, y \in R^{n}$, the inner product of $x$ and $y$ is denoted by $x \cdot y$. The Euclidean norm of any $x \in R^{n}$ is denoted by $\|x\|$, i.e., $\|x\|=\sqrt{x \cdot x}$. For any subset $U$ of $R^{n}$, int $U$ denotes the interior of $U$ in $R^{n}$ and co $U$ denotes the convex hull of $U$. A function $f: I \rightarrow R^{n}$ defined on a closed interval $I \subset R$ to $R^{n}$ is called absolutely continuous if the restriction of $f$ on any compact interval is absolutely continuous in the usual sense. The derivative of $f$ is denoted by $\dot{f}$. For any concave (or convex) function $f: U \rightarrow R \cup\{-\infty,+\infty\}$ defined on a convex subset $U$ of $R^{n}$, symbol $\partial f(x)$ denotes the set of all subgradients of function $f$ at $x \in U$, i.e., $\partial f(x)=\{p \in$ $R^{n} \mid f(x)-p \cdot x \geqq$ (or resp. $\left.\leqq\right) ~ f(y)-p \cdot y$ for all $\left.y \in U\right\}$.

A mapping $F: U \rightarrow 2^{R^{\prime}}$ defined on a subset $U$ of $R^{n}$ to the family of all non-empty subsets of $R^{\prime}$ is called a correspondence. Correspondence $F$ is called lower semi-continuous at $x_{0} \in U$ if for any $y_{0} \in F\left(x_{0}\right)$ and any sequence $\left\{x_{i}\right\}_{i \in N}$ in $U$ converging to $x_{0}$, there exists a sequence $\left\{y_{i}\right\}_{i \in N}$ converging to $y_{0}$ such that $y_{i} \in F\left(x_{i}\right)$ for all $i \in N$. Correspondence $F$ is called lower semi-continuous if $F$ is lower semi-continuous at all $x \in U$.

\section{THE MODEL}

Let $m \in N$ be the number of different commodities (capital goods) in the economy. The technology of the economy is described by a correspondence $Y:[0, \infty) \rightarrow 2^{R^{\prime \prime \prime} \times R^{\prime \prime \prime}}$. The notation $(x, y) \in Y(t)$ means that at time $t$ if we have amount $x$ of commodities (capital goods), we can increase the amount of the commodities by $y$. Namely, the pair $(x, y)$ is a technologically possible combination of the amount of capital stock and the level of investment at time $t$. Let $G_{Y}$ denote the "graph" of correspondence $Y$, i.e., $G_{Y}=\left\{(x, y, t) \in R^{m} \times R^{m} \times\right.$ $[0, \infty) \mid(x, y) \in Y(t)\}$. Also, define a correspondence $X:[0, \infty) \rightarrow 2^{R^{\prime \prime}}$ by $X(t)=$ $\left\{x \in R^{m} \mid(x, y) \in Y(t)\right.$ for some $\left.y \in R^{m}\right\}$. 
Social welfare at any point in time is represented by the instantaneous utility function $u: G_{Y} \rightarrow R$. Namely, for each $(x, y, t) \in G_{Y}, u(x, y, t)$ is interpreted as the maximum level of social satisfaction that can be attained at time $t$ if the amount of capital stock is $x$ and the level of investment is $y$.

Assumption 1: (i) The correspondence $Y$ is convex-valued, i.e.. for each $t \in[0, \infty), Y(t)$ is a convex subset of $R^{m} \times R^{m}$. (ii) For each $t \in[0, \infty)$, int $X(t)$ $\neq \varnothing$. (iii) The function $u$ is a measurable function such that, for each $t \in[0, \infty)$, $u(x, y, t)$ is a concave function of $(x, y)$ on $Y(t)$.

RIMARK 3.1: Allowing $u(x, y, t)$ to assume the value $-\infty$ on the boundary of $Y(t)$ (where the boundary is taken relative to the smallest affine set containing $Y(t)$ ) would not be a more general assumption since setting $u(x, y, t)$ equal to $-\infty$ is equivalent to excluding point $(x, y)$ from $Y(t)$. We can always perform this latter operation because $Y(t)$ is not necessarily closed. Note that such an operation does not destroy the convexity of $Y(t)$ because of the concavity of $u(x, y, t)$.

An absolutely continuous function $f:\left[t^{\prime}, t^{\prime \prime}\right] \rightarrow R^{m}$, where $t^{\prime}, t^{\prime \prime} \in[0, \infty)$ and $t^{\prime}<t^{\prime \prime}$, is called a feasible arc if $(f(t), \dot{f}(t)) \in Y(t)$ for almost every $t \in\left[t^{\prime}, t^{\prime \prime}\right]$.

Assimption 2: There exists a countable family of feasible arcs, say $\mathbb{F}$, having the following properties. (i) For each $t \in[0, \infty)$, define

$$
\begin{aligned}
\mathbb{F}_{1}=\left\{f \in \mathbb{F} \mid f \text { is a function defined on }\left[t^{\prime}, t^{\prime \prime}\right] \text { to } R^{m}\right. \\
\text { such that } \left.t^{\prime} \leqq t<t^{\prime \prime}\left(t^{\prime}=t \text { only when } t=0\right)\right\}
\end{aligned}
$$

and

$$
D_{t}=\left\{(f(t), \dot{f}(t)) \in R^{m} \times R^{m} \mid f \in \mathbb{F}_{1}\right\} .
$$

Then, $D_{t}$ is a dense subset of $Y(t)$ for all $t \in[0, \infty)$.

(ii) $\left|t^{\prime \prime} u(f(t), \dot{f}(t), t) d t\right|<+\infty$ for any $f:\left[t^{\prime}, t^{\prime \prime}\right] \rightarrow R^{m}$ in $\mathbb{F}$.

This assumption is very general, but it might be difficult to examine whether a certain model satisfies the assumption. Therefore, we shall give an assumption. which is much stronger, but seems more useful.

Assumption 2': (i) The correspondence $Y$ is lower semi-continuous. (ii) If $f:\left[t^{\prime} \cdot t^{\prime \prime}\right] \rightarrow R^{\prime \prime \prime}$ is a feasible arc whose derivative $\dot{f}$ is continuous, then $\mid f^{\prime \prime} u(f(t)$, $\dot{f}(t), l) d t \mid<+\infty$.

RIMARK 3.2: In the Appendix we shall prove that Assumption 2 is implied by Assumption 2' under Assumption 1. The proof is done by using a continuous selection theorem of Michael [11. Theorem 3.1"'] and a theorem on the existence 
of solutions for ordinary differential equations. In addition, if $u: G_{Y} \rightarrow R$ is a continuous function, then Assumption 2' (ii) is automatically satisfied. Therefore, Assumption 2 holds if correspondence $Y$ is lower semi-continuous and function $u$ is continuous.

An absolutely continuous function $k:\left[t^{\prime}, \infty\right) \rightarrow R^{m}$, where $t^{\prime} \in[0, \infty)$, is called a feasible path from time $t^{\prime}$ if $(k(t), \dot{k}(t)) \in Y(t)$ for almost every $t \in\left[t^{\prime}, \infty\right)$. For each $(x, t) \in R^{m} \times[0, \infty)$, let $A(x, t)$ denote the set of all feasible paths $k$ from time $t$ with $k(t)=x$.

Assumption 3: If $k$ is a feasible path from time $t^{\prime}$, then

$$
\int_{t^{\prime}}^{t^{\prime \prime}} u(k(t), \dot{k}(t), t) d t<+\infty \quad \text { for all } \quad t^{\prime \prime} \in\left[t^{\prime}, \infty\right) .
$$

The above assumption enables us to define a criterion of optimality for feasible paths. A feasible path $k$ from time $t^{\prime}$ is said to be overtaken by another feasible path $k^{\prime} \in A\left(k\left(t^{\prime}\right), t^{\prime}\right)$ if there exist $\epsilon>0$ and $t_{0} \geqq t^{\prime}$ such that $\int_{t^{\prime}}^{t^{\prime \prime}} u\left(k^{\prime}(t)\right.$, $\left.\dot{k}^{\prime}(t), t\right) d t>\int_{t^{\prime}}^{t^{\prime \prime}} u(k(t), \dot{k}(t), t) d t+\epsilon$ for all $t^{\prime \prime} \geqq t_{0}$.

A feasible path $k$ from time $t^{\prime}$ is called an optimal path from time $t^{\prime}$ if $k$ is not overtaken by any $k^{\prime} \in A\left(k\left(t^{\prime}\right), t^{\prime}\right)$.

REMARK 3.3: The above optimality criterion was originally introduced by von Weizsacker [18] and Gale [5]. An optimal path as defined here is commonly cailed a "weakly maximal" path by Brock [3] and McKenzie [10]. In this paper we shall not discuss the existence of optimal paths. In some specific models, the existence of optimal paths has been proved (see, for example, Brock and Haurie [4] and Takekuma [17]).

\section{THE SUPPORT PRICE THEOREM}

For each optimal path $k$ from time 0 , we can define a function $\bar{u}: G_{Y} \rightarrow R$ by

$$
\bar{u}(x, y, t)=u(x, y, t)-u(k(t), \dot{k}(t), t) .
$$

If we assume that $\int_{t^{\prime}}^{t^{\prime \prime}} u(k(t), \dot{k}(t), t) d t>-\infty$ for all $t^{\prime}, t^{\prime \prime} \in[0, \infty)$ with $t^{\prime} \leqq t^{\prime \prime}$, then we can define a function $V: R^{m} \times[0, \infty) \rightarrow R \cup\{-\infty,+\infty\}$ by

$$
V\left(x, t^{\prime}\right)=\sup _{k^{\prime} \in A\left(x, t^{\prime}\right)}\left[\liminf _{t^{\prime \prime} \rightarrow \infty} \int_{t^{\prime}}^{t^{\prime \prime}} \bar{u}\left(k^{\prime}(t), \dot{k}^{\prime}(t), t\right) d t\right] .
$$

Here we should note that functions $\bar{u}$ and $V$ are defined for a particular optimal path from time 0 , and that they depend on the optimal path. We can easily check that for each $t \in[0, \infty), \bar{u}(x, y, t)$ is a concave function of $(x, y)$ and $V(x, t)$ is a concave function of $x$. The function $\bar{u}$ can be called the normalized 
utility function with respect to the optimal path $k$. Also, the function $V$ can be called the normalized value function with respect to the optimal path $k$, which is a generalization of the usual value function defined under the assumption of finiteness of the utility integral over the infinite horizon for all feasible paths.

From now on, we shall consider an optimal path $k$ from time 0 satisfying the following assumption.

Assumption 4: (i) $\int_{t^{\prime \prime}}^{t^{\prime \prime}} u(k(t), \dot{k}(t), t) d t>-\infty$ for all $t^{\prime}, t^{\prime \prime} \in[0, \infty)$ with $t^{\prime} \leqq t^{\prime \prime}$. (ii) $k(t) \in$ int $X(t)$ for all $t \in[0, \infty)$. (iii) $\partial V(k(0), 0) \neq \varnothing$, where $\partial V(k(0), 0)$ denotes the set of all subgradients of function $V(\cdot, 0)$ at $k(0)$.

Condition (i) of this assumption together with Assumption 3 means that the optimal path $k$ generates only finite amount of utility in finite period. Condition (ii) means that the state $k(t)$ of the optimal path remains in the interior of the technology, but the control $\dot{k}(t)$ does not necessarily do so. Condition (iii) is an assumption on the initial capital stock $k(0)$ and is a necessary condition for the existence of a support price path for the optimal path $k$.

The normalized utility function $\bar{u}$ has the same properties that the original utility function $u$ has. In fact, Assumptions 1 (iii), 2 (ii), 2' (ii), 3, and 4 (i) also hold for the normalized utility function $\bar{u}$. Moreover, the normalization does not affect the optimality criterion. Thus, without loss of generality, we can identify the normalized utility function $\bar{u}$ with the original utility function $u$. From now on, when we consider a particular optimal path, we shall regard the original utility function as the normalized utility function with respect to the optimal path.

REMARK 4.1: If $f:\left[t^{\prime}, t^{\prime \prime}\right] \rightarrow R^{m}$ is a feasible arc, then

$$
V\left(f\left(t^{\prime}\right), t^{\prime}\right) \geqq \int_{t^{\prime}}^{t^{\prime \prime}} u(f(t), \dot{f}(t), t) d t+V\left(f\left(t^{\prime \prime}\right), t^{\prime \prime}\right)
$$

provided that the right-hand side of the above inequality is well-defined.

MaIn TheOREM: Let $k$ be an optimal path from time 0 satisfying Assumption 4. Then, under Assumption 1, 2 (or $\left.2^{\prime}\right)$, and 3 , for any $p \in \partial u(k(0), 0)$ there exists an absolutely continuous function $q:[0, \infty) \rightarrow R^{m}$ with the following properties:

$$
\begin{aligned}
& q(0)=p . \\
& q(t) \in \partial V(k(t), t) \quad \text { for all } t \in[0, \infty) . \\
& -(\dot{q}(t), q(t)) \in \partial u(k(t), \dot{k}(t), t) \quad \text { for almost every } \quad t \in[0, \infty) .
\end{aligned}
$$

In the above, for each $t \in[0, \infty)$, symbols $\partial V(\cdot, t)$ and $\partial u(\cdot, \cdot, t)$ denote the sets of all subgradients for functions $V(\cdot, t)$ and $u(\cdot, \cdot, t)$, respectively. 
A proof of this theorem will be given later. The theorem presented here is a counterpart of the theorem which was proved by McKenzie [10, L.1] in a discrete time model. There are some new features in our theorem which are not found in the usual duality theory for continuous time models. First, we have replaced the usual assumption of finiteness of the utility integral over the infinite horizon for all feasible paths by the weaker set, Assumptions 3 and 4 (i), (iii).

Second, condition (i) of our theorem says that we can choose any point in $\partial V(k(0), 0)$ as an initial price for the support price path. That is, for any point in $\partial V(k(0), 0)$, there exists a price path which starts from the point and supports the optimal path.

Third, the theorem says that conditions (ii) and (iii) hold at the same time. In other words, the price path $q$ supports the value function $V(\cdot, t)$ as well as the utility function $u(\cdot, \cdot, t)$ at every time $t$. The existence of a price path with such a property is not obvious in non-differentiable models.

Define a function $H: R^{m} \times R^{m} \times[0, \infty) \rightarrow R \cup\{-\infty,+\infty\}$ by

$$
H(p, x, t)=\sup \{u(x, y, t)+p \cdot y \mid(x, y) \in Y(t)\} .
$$

Function $H$ is commonly called the Hamiltonian function. It is well known that, under Assumption l (i), (iii), for each $t \in[0, \infty) H(p, x, t)$ is a convex function of $p$ and is a concave function of $x$.

Remark 4.2: Condition (iii) of the Main Theorem is equivalent to the following: $\dot{k}(t) \in \partial_{1} H(q(t), k(t), t), \quad-\dot{q}(t) \in \partial_{2} H(q(t), k(t), t)$, and $H(q(t), k(t), t)$ $=u(k(t), \dot{k}(t), t)+q(t) \cdot \dot{k}(t)$ for almost every $t \in[0, \infty)$. Here, for each $t \in$ $[0, \infty)$, symbols $\partial_{1} H(\cdot, k(t), t)$ and $\partial_{2} H(q(t), \cdot, t)$ denote the sets of all subgradients for functions $H(\cdot, k(t), t)$ and $H(q(t), \cdot, t)$ respectively. Although the proof of equivalence is not entirely trivial, we shall not include it since the equivalence is a well-known fact.

\section{THE OUTLINE OF THE PROOF OF THE MAIN THEOREM}

Let $k$ be an optimal path from time 0 in the Main Theorem. An absolutely continuous function $g:[r, s] \rightarrow R^{m}$, where $r, s \in[0, \infty)$ with $r<s$, is said to be $a$ support price arc for $k$ if $g(t) \in \partial V(k(t), t)$ for all $t \in[r, s]$ and $-(\dot{g}(t), g(t))$ $\in \partial u(k(t), \dot{k}(t), t)$ for almost every $t \in[r, s]$.

AuXILIARY Theorem: For each $t_{0} \in[0, \infty)$ there exists a pair of numbers $\{r, s\}$ with $r \leqq t_{0}<s\left(r=t_{0}\right.$ only when $\left.t_{0}=0\right)$ such that for any $p_{0} \in \partial V(k(r), r)$ there exists a support price arc for $k, g:[r, s] \rightarrow R^{m}$, with $g(r)=p_{0}$.

This Auxiliary Theorem may be called "the local existence theorem of a support price path." We shall show that the Main Theorem is implied by the Auxiliary Theorem.

Consider an interval $[0,1]$. Since $[0,1]$ is compact, the Auxiliary Theorem implies that there exist finitely many pairs of numbers $\left\{r_{i}, s_{i}\right\}_{i=1}^{\prime}$ with $[0,1]$ 
$\subset \bigcup_{i=1}^{\prime}\left[r_{i}, s_{i}\right]$ such that each pair $\left\{r_{i}, s_{i}\right\}$ has the same desirable property that the pair $\{r, s\}$ in the Auxiliary Theorem has. Without loss of generality, we can assume that $r_{1}=0, r_{i}<s_{i-1}<r_{i+1}<s_{i}(i=2, \ldots, l-1)$, and $s_{l}=1$. Then, since $r_{1}=0$ and $p \in \partial V(k(0), 0)$, there exists a support price arc for $k, g_{1}:\left[r_{1}, s_{1}\right]$ $\rightarrow R^{m}$, with $g_{1}\left(r_{1}\right)=p$. Since $r_{2}<s_{1}$ and $g_{1}\left(r_{2}\right) \in \partial V\left(k\left(r_{2}\right), r_{2}\right)$, there exists a support price arc for $k, g_{2}:\left[r_{2}, s_{2}\right] \rightarrow R^{m}$, with $g_{2}\left(r_{2}\right)=g_{1}\left(r_{2}\right)$. Continue this procedure, and we have support price arcs for $k, g_{1}, g_{2}, \ldots, g_{l}$ such that $g_{i}\left(r_{i+1}\right)=g_{i+1}\left(r_{i+1}\right)$ for all $i$ with $1 \leqq i \leqq l-1$. Define a function $q_{1}:[0,1] \rightarrow R^{m}$ by $q(t)=g_{i}(t)$ when $t \in\left[r_{i}, r_{i+1}\right]$ for some $i$, and by $q(t)=g_{l}(t)$ when $t \in\left[r_{l}, s_{l}\right]$. Then, by construction, $q_{1}$ is a support price arc for $k$ with $q_{1}(0)=p$.

In the exact same way, since $q_{1}(1) \in \partial V(k(1), 1)$, we can construct a support price arc for $k, q_{2}:[1,2] \rightarrow R^{m}$, with $q_{2}(1)=q_{1}(1)$. By repeating this procedure, for each $n \in N$ we have a support price arc for $k, q_{n}:[n-1, n] \rightarrow R^{m}$ such that $q_{n}(n)=q_{n+1}(n)$ for all $n \in N$. Define a function $q:[0, \infty) \rightarrow R^{m}$ by $q(t)=q_{n}(t)$ when $t \in[n-1, n]$ for some $n$. Obviously, by construction, function $q$ satisfies all the conditions required in the Main Theorem.

Hence, we have proved that the Main Theorem is implied by the Auxiliary Theorem, and all that remains to be done is to prove the Auxiliary Theorem.

\section{PROOF OF THE AUXILIARY THEOREM}

To prove the Auxiliary Theorem, let $k$ be an optimal path from time 0 and pick $t_{0} \in[0, \infty)$.

LeMma 6.1: There exist a pair of numbers $\{r, s\}$ with $r \leqq t_{0}<s\left(r=t_{0}\right.$ only when $\left.t_{0}=0\right)$ and $m+1$ feasible arcs $h_{i}:[r, s] \rightarrow R^{m}(i=0,1, \ldots, m)$ having the following properties: (i) $k(t) \in$ int $\operatorname{co}\left\{h_{0}(t), h_{1}(t), \ldots, h_{m}(t)\right\}$ for all $t \in[r, s]$, (ii) $\left|\int_{r}^{s} u\left(h_{i}(t), \dot{h}_{i}(t), t\right) d t\right|<+\infty$ for all $i$.

ProOf: Since $k\left(t_{0}\right) \in$ int $X\left(t_{0}\right)$ by Assumption 4 (ii), from the family $\mathbb{F}_{t_{0}}$ in Assumption 2 (i) we can choose $m+1$ feasible arcs, say $f_{0}, f_{1}, \ldots, f_{m}$ such that $k\left(t_{0}\right) \in$ int $\operatorname{co}\left\{f_{0}\left(t_{0}\right), f_{1}\left(t_{0}\right), \ldots, f_{m}\left(t_{0}\right)\right\}$. Since functions, $f_{0}, f_{1}, \ldots, f_{m}$, and $k$ are continuous, there is a pair of numbers $\{r, s\}$ with $r \leqq t_{0}<s\left(r=t_{0}\right.$ only when $t_{0}=0$ ) such that

$$
k(t) \in \operatorname{int} \operatorname{co}\left\{f_{0}(t), f_{1}(t), \ldots, f_{m}(t)\right\} \quad \text { for all } t \in[r, s] .
$$

For each $i$, let $h_{i}$ be the restriction of $f_{i}$ on $[r, s]$. Then functions, $h_{0}, h_{1}, \ldots, h_{m}$ satisfy condition (i) of this lemma. Also, by virtue of Assumption 2 (ii), we know that each $h_{i}$ satisfies Condition (ii).

Q.E.D.

To prove that the pair of numbers $\{r, s\}$ in this lemma has the desirable property required in the Auxiliary Theorem, we need the following lemma, which will play a most important role in our argument. The lemma corresponds to the "induction" procedure used by Weitzman [20] and McKenzie [9 and 10] in discrete time models. 
Lemma 6.2: For any $t^{\prime}, t^{\prime \prime} \in[r, s]$ with $t^{\prime}<t^{\prime \prime}$ and any $p^{\prime} \in \partial V\left(k\left(t^{\prime}\right), t^{\prime}\right)$, there exists $p^{\prime \prime} \in \partial V\left(k\left(t^{\prime \prime}\right), t^{\prime \prime}\right)$ such that

$$
\begin{aligned}
& \int_{t^{\prime}}^{t^{\prime \prime}} u(k(t), \dot{k}(t), t) d t-p^{\prime} \cdot k\left(t^{\prime}\right)+p^{\prime \prime} \cdot k\left(t^{\prime \prime}\right) \\
& \quad \geqq \int_{t^{\prime}}^{t^{\prime \prime}} u(f(t), \dot{f}(t), t) d t-p^{\prime} \cdot f\left(t^{\prime}\right)+p^{\prime \prime} \cdot f\left(t^{\prime \prime}\right)
\end{aligned}
$$

for all feasible arc $f:\left[t^{\prime}, t^{\prime \prime}\right] \rightarrow R^{m}$ with $\left|\int_{t^{\prime}}^{t^{\prime \prime}} u(f(t), \dot{f}(t), t) d t\right|<+\infty$.

Proof: By definition of the value function $V$ (see Remark 4.1),

$$
V\left(f\left(t^{\prime}\right), t^{\prime}\right) \geqq \int_{t^{\prime}}^{t^{\prime \prime}} u(f(t), \dot{f}(t), t) d t+V\left(f\left(t^{\prime \prime}\right), t^{\prime \prime}\right)
$$

for all feasible arc $f:\left[t^{\prime}, t^{\prime \prime}\right] \rightarrow R^{m}$, provided that the right-hand side of the above inequality is well-defined. Also, $p^{\prime} \in \partial V\left(k\left(t^{\prime}\right), t^{\prime}\right)$ implies

$$
V\left(k\left(t^{\prime}\right), t^{\prime}\right)-p^{\prime} \cdot k\left(t^{\prime}\right) \geqq V\left(x, t^{\prime}\right)-p^{\prime} \cdot x \quad \text { for all } \quad x \in R^{m} .
$$

Since $V\left(k\left(t^{\prime}\right), t^{\prime}\right)=\int_{t^{\prime \prime}}^{t^{\prime \prime}} u(k(t), \dot{k}(t), t) d t+V\left(k\left(t^{\prime \prime}\right), t^{\prime \prime}\right)$, by taking $x=f\left(t^{\prime}\right)$ in the above two inequalities we have

$$
\begin{aligned}
& \int_{t^{\prime}}^{t^{\prime \prime}} u(k(t), \dot{k}(t), t) d t+V\left(k\left(t^{\prime \prime}\right), t^{\prime \prime}\right)-p^{\prime} \cdot k\left(t^{\prime}\right) \\
& \quad \geqq \int_{t^{\prime}}^{t^{\prime \prime}} u(f(t), \dot{f}(t), t) d t+V\left(f\left(t^{\prime \prime}\right), t^{\prime \prime}\right)-p^{\prime} \cdot f\left(t^{\prime}\right)
\end{aligned}
$$

for all feasible arc $f:\left[t^{\prime}, t^{\prime \prime}\right] \rightarrow R^{m}$, provided that the right-hand side of the above inequality is well-defined.

Let $\alpha_{*}$ denote the left-hand side of inequality (6.1). Define two subsets $C_{1}$ and $C_{2}$ of $R^{m+1}$ by

$$
\begin{gathered}
C_{1}=\left\{(\alpha, x) \in R \times R^{m} \mid \alpha>\alpha_{*}-\int_{t^{\prime}}^{t^{\prime \prime}} u(f(t), \dot{f}(t), t) d t+p^{\prime} \cdot f\left(t^{\prime}\right)\right. \\
\text { and } \left.\quad x=f\left(t^{\prime \prime}\right) \text { for some feasible arc } f:\left[t^{\prime}, t^{\prime \prime}\right] \rightarrow R^{m}\right\},
\end{gathered}
$$

and

$$
C_{2}=\left\{(\alpha, x) \in R \times R^{m} \mid \alpha \leqq V\left(x, t^{\prime \prime}\right)\right\} .
$$

We can easily check that both $C_{1}$ and $C_{2}$ are non-empty and convex. Also, from (6.1), it follows that they are disjoint. Therefore, by a well-known separation theorem, we have a non-zero vector $\left(\pi,-p^{\prime \prime}\right) \in R \times R^{m}$ such that $\pi \alpha-p^{\prime \prime} \cdot x$ $\geqq \pi \alpha^{\prime}-p^{\prime \prime} \cdot x^{\prime}$ for all $(\alpha, x) \in C_{1}$ and $\left(\alpha^{\prime}, x^{\prime}\right) \in C_{2}$. Since point $\left(V\left(k\left(t^{\prime \prime}\right), t^{\prime \prime}\right)\right.$, $\left.k\left(t^{\prime \prime}\right)\right)$ in $C_{2}$ belongs to the boundary of $C_{1}$, it follows that

$$
\pi \alpha-p^{\prime \prime} \cdot x \geqq \pi V\left(k\left(t^{\prime \prime}\right), t^{\prime \prime}\right)-p^{\prime \prime} \cdot k\left(t^{\prime \prime}\right) \geqq \pi \alpha^{\prime}-p^{\prime \prime} \cdot x^{\prime}
$$


for all $(\alpha, x) \in C_{1}$ and all $\left(\alpha^{\prime}, x^{\prime}\right) \in C_{2}$. The first inequality of (6.2) implies that

$$
\begin{aligned}
& \pi\left[\int_{t^{\prime}}^{t^{\prime \prime}} u(k(t), \dot{k}(t), t) d t-p^{\prime} \cdot k\left(t^{\prime}\right)\right]+p^{\prime \prime} \cdot k\left(t^{\prime \prime}\right) \\
& \quad \geqq \pi\left[\int_{t^{\prime}}^{t^{\prime \prime}} u(f(t), \dot{f}(t), t) d t-p^{\prime} \cdot f\left(t^{\prime}\right)\right]+p^{\prime \prime} \cdot f\left(t^{\prime \prime}\right)
\end{aligned}
$$

for all feasible arc $f:\left[t^{\prime}, t^{\prime \prime}\right] \rightarrow R^{m}$ with $\left|\int_{t^{\prime}}^{t^{\prime \prime}} u(f(t), \dot{f}(t), t) d t\right|<+\infty$.

We can easily show that the particular forms of $C_{1}$ and $C_{2}$ imply that $\pi \geqq 0$. Suppose that $\pi=0$. Then, it follows from (6.3) that $p^{\prime \prime} \cdot k\left(t^{\prime \prime}\right) \geqq p^{\prime \prime} \cdot h_{i}\left(t^{\prime \prime}\right)$ for all $i$, where $h_{0}, h_{1}, \ldots, h_{m}$ are feasible arcs satisfying condition (i) of Lemma 6.1. Therefore, $p^{\prime \prime}=0$. However, this is a contradiction to the premise that $\left(\pi,-p^{\prime}\right)$ $\neq 0$. Thus, $\pi>0$. Hence, the second inequality of (6.2) implies that

$$
\pi V\left(k\left(t^{\prime \prime}\right), t^{\prime \prime}\right)-p^{\prime \prime} \cdot k\left(t^{\prime \prime}\right) \geqq \pi V\left(x, t^{\prime \prime}\right)-p^{\prime \prime} \cdot x \quad \text { for all } \quad x \in R^{m} .
$$

Without loss of generality, we can assume that $\pi=1$. Therefore, (6.4) implies that $p^{\prime \prime} \in \partial V\left(k\left(t^{\prime \prime}\right), t^{\prime \prime}\right)$. Hence, this lemma follows from (6.3).

Q.E.D.

Pick $p_{0} \in \partial V(k(r), r)$ and let us begin to prove that the pair $\{r, s\}$ in Lemma 6.1 has the desirable property required in the Auxiliary Theorem. For each $n \in N$, define a finite subset $T_{n}$ of $[r, s]$ by

$$
T_{n}=\left\{t \in[r, s] \mid t=r+i(s-r) / 2^{n} \text { and } i=0,1, \ldots, 2^{n}\right\} .
$$

Lemma 6.3: For each $n \in N$, there exists a set of $2^{n}+1$ vectors in $R^{m}$, say $\left\{p_{n}(t) \mid t \in T_{n}\right\}$, having the following properties:

$$
\begin{aligned}
& \text { (i) } \quad p_{n}(r)=p_{0}, \\
& \text { (ii) } \quad p_{n}(t) \in \partial V(k(t), t) \quad \text { for all } t \in T_{n}, \\
& \text { (iii) } \quad \int_{t^{\prime}}^{t^{\prime \prime}} u(k(t), \dot{k}(t), t) d t-p_{n}\left(t^{\prime}\right) \cdot k\left(t^{\prime}\right)+p_{n}\left(t^{\prime \prime}\right) \cdot k\left(t^{\prime \prime}\right) \\
& \geqq \int_{t^{\prime}}^{t^{\prime \prime}} u(f(t), \dot{f}(t), t) d t-p_{n}\left(t^{\prime}\right) \cdot \dot{f}\left(t^{\prime}\right)+p_{n}\left(t^{\prime \prime}\right) \cdot f\left(t^{\prime \prime}\right)
\end{aligned}
$$

for all feasible arc $f:\left[t^{\prime}, t^{\prime \prime}\right] \rightarrow R^{m}$ with $\left|\int_{t^{\prime}}^{t^{\prime \prime}} u(f(t), \dot{f}(t), t) d t\right|<+\infty$, where $t^{\prime}, t^{\prime \prime}$ $\in T_{n}$.

Proof: For each $n \in N$, apply Lemma 6.2 successively to $2^{n}$ pairs of numbers, $\left\{r, r+(s-r) / 2^{n}\right\},\left\{r+(s-r) / 2^{n}, r+2(s-r) / 2^{n}\right\}, \ldots,\left\{r+i(s-r) / 2^{n}, r+\right.$ $\left.(i+1)(s-r) / 2^{n}\right\}, \ldots,\left\{r+\left(2^{n}-1\right)(s-r) / 2^{n}, s\right\}$. Then, we have a set of $2^{n}+1$ vectors, say $\left\{p_{n}(t) \mid t \in T_{n}\right\}$, which has the desirable property in this lemma. Q.E.D.

Lemma 6.4: $\left\{p_{n}(t) \mid t \in T_{n}\right.$ and $\left.n \in N\right\}$ is a bounded subset of $R^{m}$. 
Proof: Suppose that this lemma were not true. Then, there is an infinite subset $N_{0}$ of $N$ such that for each $n \in N_{0}$ we can choose an element from $T_{n}$, say $t_{n}$, and $\left\|p_{n}\left(t_{n}\right)\right\|$ goes to $+\infty$ as $n$ goes to $+\infty$. Without loss of generality, we can assume that $\lim _{n \rightarrow x} t_{n}=\underline{t}$ and $\lim _{n \rightarrow \infty}\left(p_{n}\left(t_{n}\right) /\left\|p_{n}\left(t_{n}\right)\right\|\right)=\underline{p} \neq 0$. On the other hand. by Lemmas 6.1 and 6.3 (iii), for all $h_{i}$ and for all $n \in N_{0}$.

$$
\begin{aligned}
& \frac{1}{\left\|p_{n}\left(t_{n}\right)\right\|}\left[\int_{r}^{t_{n}} u(k(t), \dot{k}(t), t) d t-p_{0} \cdot k(r)+p_{n}\left(t_{n}\right) \cdot k\left(t_{n}\right)\right] \\
& \quad \geqq \frac{1}{\left\|p_{n}\left(t_{n}\right)\right\|}\left[\int_{r}^{t_{n}} u\left(h_{i}(t), \dot{h}_{i}(t), t\right) d t-p_{0} \cdot h_{i}(r)+p_{n}\left(t_{n}\right) \cdot h_{i}\left(t_{n}\right)\right] .
\end{aligned}
$$

Therefore, in the limit, $\underline{p} \cdot k(\underline{t}) \geqq \underline{p} \cdot h_{i}(\underline{t})$ for all $h_{i}$. Thus, by Lemma 6.1 (i), we can conclude that $\underline{p}=0$. This is a contradiction.

Lemma 6.5: Let $T=\cup_{n \in N} T_{n}$. Then, there exists a bounded function $g_{0}: T$ $\rightarrow R^{\prime \prime}$ having the following properties:

$$
\begin{aligned}
& g_{0}(r)=p_{0}, \\
& g_{0}(t) \in \partial V(k(t), t) \quad \text { for all } \quad t \in T, \\
& \int_{t^{\prime}}^{t^{\prime \prime}} u(k(t), \dot{k}(t), t) d t-g_{0}\left(t^{\prime}\right) \cdot k\left(t^{\prime}\right)+g_{0}\left(t^{\prime \prime}\right) \cdot k\left(t^{\prime \prime}\right) \\
& \quad \geqq \int_{t^{\prime}}^{t^{\prime \prime}} u(f(t), \dot{f}(t), t) d t-g_{0}\left(t^{\prime}\right) \cdot f\left(t^{\prime}\right)+g_{0}\left(t^{\prime \prime}\right) \cdot f\left(t^{\prime \prime}\right)
\end{aligned}
$$

for all feasible arc $f:\left[t^{\prime}, t^{\prime \prime}\right] \rightarrow R^{m}$ with $\left|\int_{t^{\prime}}^{t^{\prime \prime}} u(f(t), \dot{f}(t), t) d t\right|<+\infty$, where $t^{\prime}, t^{\prime \prime}$ $\in T$.

Proof: By Lemma 6.3, for each $t \in T_{1}$ we have a sequence $\left\{p_{n}(t) \mid n \in N\right\}$. Since $T_{1}$ is a finite set, by Lemma 6.4 we can find an infinite subset of $N$, say $N_{1}$, such that for any $t \in T_{1}$, sequence $\left\{p_{n}(t) \mid n \in N_{1}\right\}$ converges to a point, say $g_{0}(t)$. Next, by Lemma 6.3, for each $t \in T_{2}$ we have a sequence $\left\{p_{n}(t) \mid n \in N_{1}\right.$ with $n \geqq 2\}$. Again, since $T_{2}$ is a finite set, by Lemma 6.4 we have an infinite subset of $N_{1}$, say $N_{2}$, such that for any $t \in T_{2}$, sequence $\left\{p_{n}(t) \mid n \in N_{2}\right\}$ converges to a point, say $g_{0}(t)$. (Although $T_{1} \subset T_{2}$, this notation is consistent since $N_{1} \supset N_{2}$.) By repeating this procedure, we have sets, $N_{1}, N_{2}, N_{3}, \ldots$ with $N \supset N_{1} \supset N_{2} \supset N_{3} \supset, \ldots$ such that for any $i$ and any $t \in T_{i}$, sequence $\left\{p_{n}(t) \mid n\right.$ $\left.\in N_{i}\right\}$ converges to a point, say $g_{0}(t)$. In this way we can define a function $g_{0}: T \rightarrow R^{m}$, which is clearly bounded because of Lemma 6.4.

Obviously, condition (i) of this lemma is implied by Lemma 6.3 (i). Also, if $t \in T$, i.e., $t \in T_{i}$ for some $i$, then $t \in T_{n}$ for all $n \in N_{i}$. Therefore condition (ii) of Lemma 6.3 holds for all $n \in N_{i}$. Hence, since set $\partial V(k(t), t)$ is closed, condition (ii) of this lemma also holds in the limit. Furthermore, if $t^{\prime}, t^{\prime \prime} \in T$, and $t^{\prime}<t^{\prime \prime}$, then $t^{\prime}, t^{\prime \prime} \in T_{j}$ for some $j$. Since $t^{\prime}, t^{\prime \prime} \in T_{n}$ for all $n \in N_{j}$, condition (iii) of 
Lemma 6.3 holds for all $n \in N_{j}$. Hence condition (iii) of this lemma also holds in the limit.

Limma 6.6: Function $g_{0}$ is continuous.

Proof: Suppose that function $g_{0}$ were not continuous. Then, since function $g_{0}$ is bounded, there are two sequences $\left\{t_{n}^{\prime}\right\}_{n \in N}$ and $\left\{t_{n}^{\prime \prime}\right\}_{n \in N}$ converging to a point $\underline{t}$ such that $t_{n}^{\prime} \leqq \underline{t} \leqq t_{n}^{\prime \prime}$ for all $n \in N$ and $\lim _{n \rightarrow \infty}\left(g_{0}\left(t_{n}^{\prime \prime}\right)-g_{0}\left(t^{\prime}\right)\right)=p \neq 0$. By Lemmas 6.1 and 6.5 (iii), $p \cdot k(\underline{t}) \geqq p \cdot h_{i}(\underline{t})$ for all $h_{i}$. Therefore, by Lemma 6.1 (i), we can conclude that $\underline{p}=0$. This is a contradiction.

Q.E.D.

Limma 6.7: There exists a continuous function $g:[r, s] \rightarrow R^{m}$ having the following properties:

$$
\begin{aligned}
& g(r)=p_{0}, \\
& g(t) \in \partial V(k(t), t) \quad \text { for all } \quad t \in[r, s], \\
& \int_{t^{\prime}}^{t^{\prime \prime}} u(k(t), \dot{k}(t), t) d t-g\left(t^{\prime}\right) \cdot k\left(t^{\prime}\right)+g\left(t^{\prime \prime}\right) \cdot k\left(t^{\prime \prime}\right) \\
& \quad \geqq \int_{t^{\prime}}^{t^{\prime \prime}} u(f(t), \dot{f}(t), t) d t-g\left(t^{\prime}\right) \cdot f\left(t^{\prime}\right)+g\left(t^{\prime \prime}\right) \cdot f\left(t^{\prime \prime}\right)
\end{aligned}
$$

for all feasible arc $f:\left[t^{\prime}, t^{\prime \prime}\right] \rightarrow R^{m}$ with $\left|\int_{t^{\prime}}^{t^{\prime \prime}} u(f(t), \dot{f}(t), t) d t\right|<+\infty$, where $t^{\prime}, t^{\prime \prime}$ $\in[r, s]$.

Proof: Since $T$ is a dense subset of $[r, s]$, the bounded and continuous function $g_{0}$ can be uniquely extended to a continuous function, say $g:[r, s]$ $\rightarrow R^{m}$. We shall show that function $g$ has the desirable properties.

Condition (i) of this lemma immediately follows from condition (i) of Lemma 6.5. Also, since function $g$ is continuous extension of function $g_{0}$ and since $T$ is dense in $[r, s]$, condition (iii) of this lemma immediately follows from condition (iii) of Lemma 6.5. To prove condition (ii) of this lemma, let $t \in[r, s]$ and assume that $t \notin T$. Since $T$ is dense in $[r, s]$, we have a sequence $\left\{t_{n}\right\}_{n \in N}$ in $T$ converging to $t$ such that $t_{n} \leqq t$ for all $n \in N$. Let $f \in \mathbb{F}_{t}$, where $\mathbb{F}_{t}$ is a family in Assumption 2. Then, by Lemma 6.5 (ii), $V\left(k\left(t_{n}\right), t_{n}\right)-g_{0}\left(t_{n}\right) \cdot k\left(t_{n}\right) \geqq V\left(f\left(t_{n}\right)\right.$, $\left.t_{n}\right)-g_{0}\left(t_{n}\right) \cdot f\left(t_{n}\right)$ for all sufficiently large $n \in N$. Namely, by definition of the value function $V$,

$$
\begin{aligned}
& \int_{t_{n}}^{t} u(k(\tau), \dot{k}(\tau), \tau) d \tau+V(k(t), t)-g_{0}\left(t_{n}\right) \cdot k\left(t_{n}\right) \\
& \quad \geqq \int_{t_{n}}^{t} u(f(\tau), \dot{f}(\tau), \tau) d \tau+V(f(t), t)-g_{0}\left(t_{n}\right) \cdot f\left(t_{n}\right)
\end{aligned}
$$

for all sufficiently large $n \in N$. Therefore, in the limit, we have $V(k(t), t)-g(t) \cdot$ $k(t) \geqq V(f(t), t)-g(t) \cdot f(t)$. Thus, by Assumption $2, V(k(t), t)-g(t) \cdot k(t)$ 
$\geqq V(x, t)-g(t) \cdot x$ for all $(x, y) \in D_{t}$. This implies that $g(t) \in \partial V(k(t), t)$, because $k(t) \in$ int $X(t)$ by Assumption 4 (ii) and $D_{t}$ is dense in $Y(t)$ by Assumption 2 (i). Thus, condition (ii) of this lemma has been proved.

Q.E.D.

\section{LEMMA 6.8: Function $g$ is absolutely continuous.}

Proof: By Lemma 6.1 we can easily prove the following facts.

(6.5) There exists $\delta>0$ such that $\left\|h_{i}(t)-k(t)\right\| \geqq \delta$ for all $t \in[r, s]$ and for all $h_{i}$.

(6.6) There exists $\gamma>0$ such that if $v \in R^{m}$ and $t \in[r, s]$, then

$$
v \cdot\left(h_{i}(t)-k(t)\right) \geqq \gamma\|v\| \cdot\left\|h_{i}(t)-k(t)\right\| \text { for some } h_{i} .
$$

By Lemmas 6.1 and 6.7 (iii), for all $h_{i}$ and $t^{\prime}, t^{\prime \prime} \in[r, s]$ with $t^{\prime} \leqq t^{\prime \prime}$,

$$
\begin{aligned}
\int_{t^{\prime}}^{t^{\prime \prime}} u( & k(t), \dot{k}(t), t) d t+g\left(t^{\prime \prime}\right) \cdot\left(k\left(t^{\prime \prime}\right)-k\left(t^{\prime}\right)\right) \\
& \quad-\int_{t^{\prime}}^{t^{\prime \prime}} u\left(h_{i}(t), \dot{h_{i}}(t), t\right) d t-g\left(t^{\prime \prime}\right) \cdot\left(h_{i}\left(t^{\prime \prime}\right)-h_{i}\left(t^{\prime}\right)\right) \\
\geqq & \left(g\left(t^{\prime \prime}\right)-g\left(t^{\prime}\right)\right) \cdot\left(h_{i}\left(t^{\prime}\right)-k\left(t^{\prime}\right)\right) .
\end{aligned}
$$

Moreover, since function $g$ is continuous,

$$
\text { there exists } \beta>0 \text { such that }\|g(t)\| \leqq \beta \text { for all } t \in[r, s] \text {. }
$$

Now we can prove the following inequalities.

$$
\begin{aligned}
\frac{1}{\gamma \delta} \sum_{i=0}^{m} & {\left[\int_{t^{\prime}}^{t^{\prime \prime}}|u(k(t), \dot{k}(t), t) d t| d t+\beta\left\|k\left(t^{\prime \prime}\right)-k\left(t^{\prime}\right)\right\|\right.} \\
& \left.+\int_{t^{\prime}}^{t^{\prime \prime}}\left|u\left(h_{i}(t), \dot{h_{i}}(t), t\right)\right| d t+\beta\left\|h_{i}\left(t^{\prime \prime}\right)-h_{i}\left(t^{\prime}\right)\right\|\right] \\
\geqq & \frac{1}{\gamma \delta} \max _{0 \leqq i \leqq m}\left[\int_{t^{\prime}}^{t^{\prime \prime}} u(k(t), \dot{k}(t), t) d t+g\left(t^{\prime \prime}\right) \cdot\left(k\left(t^{\prime \prime}\right)-k\left(t^{\prime}\right)\right)\right. \\
& \left.\quad-\int_{t^{\prime}}^{t^{\prime \prime}} u\left(h_{i}(t), \dot{h_{i}}(t), t\right) d t-g\left(t^{\prime \prime}\right) \cdot\left(h_{i}\left(t^{\prime \prime}\right)-h_{i}\left(t^{\prime}\right)\right)\right] \\
\geqq & \frac{1}{\gamma \delta} \max _{0 \leqq i \leqq m}\left(g\left(t^{\prime \prime}\right)-g\left(t^{\prime}\right)\right) \cdot\left(h_{i}\left(t^{\prime}\right)-k\left(t^{\prime}\right)\right) \\
\geqq & \frac{1}{\delta} \min _{0 \leqq i \leqq m}\left\|g\left(t^{\prime \prime}\right)-g\left(t^{\prime}\right)\right\| \cdot\left\|h_{i}\left(t^{\prime}\right)-k\left(t^{\prime}\right)\right\| \\
\geqq & \left\|g\left(t^{\prime \prime}\right)-g\left(t^{\prime}\right)\right\|
\end{aligned}
$$


for all $t^{\prime}, t^{\prime \prime} \in[r, s]$ with $t^{\prime} \leqq t^{\prime \prime}$, where the first inequality is implied by (6.8), the second by (6.7), the third by (6.6), and the fourth by (6.5). Hence, since Lebesgue integrals are absolutely continuous and functions $h_{0}, h_{1}, \ldots, h_{m}, k$ are absolutely continuous, function $g$ is also absolutely continuous.

Q.E.D.

Lemma 6.9: $-(\dot{g}(t), g(t)) \in \partial u(k(t), \dot{k}(t), t)$ for almost every $t \in[r, s]$.

Proof: For almost every $t \in[r, s]$ (see, for example, Natanson [12, p. 255]),

$$
\begin{aligned}
& \lim _{\theta \rightarrow 0+} \frac{1}{\theta} \int_{t}^{t+\theta} u(k(\tau), \dot{k}(\tau), \tau) d \tau=u(k(t), \dot{k}(t), t), \\
& \lim _{\theta \rightarrow 0+} \frac{g(t+\theta)-g(t)}{\theta}=\dot{g}(t), \quad \text { and } \\
& \lim _{\theta \rightarrow 0+} \frac{k(t+\theta)-k(t)}{\theta}=\dot{k}(t) .
\end{aligned}
$$

Furthermore, since $\mathbb{F}$ in Assumption 2 is a countable family, for almost every $t \in[r, s]$,

$$
\begin{aligned}
& \lim _{\theta \rightarrow 0+} \frac{1}{\theta} \int_{t}^{t+\theta} u(f(\tau), \dot{f}(\tau), \tau) d \tau=u(f(t), \dot{f}(t), t) \quad \text { and } \\
& \lim _{\theta \rightarrow 0+} \frac{f(t+\theta)-f(t)}{\theta}=\dot{f}(t) \quad \text { for all } f \in \mathbb{F}_{t} .
\end{aligned}
$$

Let $t \in[r, s]$ be such a point in the above, and pick $f \in \mathbb{F}_{1}$. Then, for all sufficiently small $\theta>0$, we have by Lemma 6.7 (iii)

$$
\begin{aligned}
\frac{1}{\theta} \int_{t}^{t+\theta} u(k(\tau), \dot{k}(\tau), \tau) d \tau+\frac{g(t+\theta)-g(t)}{\theta} \cdot k(t) \\
\quad+g(t+\theta) \cdot \frac{k(t+\theta)-k(t)}{\theta} \\
\geqq \frac{1}{\theta} \int_{t}^{t+\theta} u(f(\tau), \dot{f}(\tau), \tau) d \tau+\frac{g(t+\theta)-g(t)}{\theta} \cdot f(t) \\
\quad+g(t+\theta) \cdot \frac{f(t+\theta)-f(t)}{\theta} .
\end{aligned}
$$

Therefore, in the limit as $\theta$ goes to 0 , we have

$$
\begin{aligned}
& u(k(t), \dot{k}(t), t)+\dot{g}(t) \cdot k(t)+g(t) \cdot \dot{k}(t) \\
& \quad \geqq u(f(t), \dot{f}(t), t)+\dot{g}(t) \cdot f(t)+g(t) \cdot \dot{f}(t) .
\end{aligned}
$$


Hence, by Assumption 2 (i),

$$
\begin{aligned}
& u(k(t), \dot{k}(t), t)+\dot{g}(t) \cdot k(t)+g(t) \cdot \dot{k}(t) \\
& \quad \geqq u(x, y, t)+\dot{g}(t) \cdot x+g(t) \cdot y \quad \text { for all } \quad(x, y) \in D_{t} .
\end{aligned}
$$

This implies that $-(\dot{g}(t), g(t)) \in \partial u(k(t), \dot{k}(t), t)$, because $D_{t}$ is dense in $Y(t)$ by Assumption 2 (i), and because $u(\cdot, \cdot, t)$ is a concave function on $Y(t)$. Q.E.D.

Obviously, Lemmas 6.7, 6.8, and 6.9 imply the Auxiliary Theorem. This completes the proof of the Auxiliary Theorem.

\section{Hitotsubashi University}

Manuscript received December, 1978; revision received July, 1980.

\section{APPENDIX}

In this Appendix we shall prove that Assumption 2 is implied by Assumption 2' under Assumption 1 .

Let $G_{X}$ denote the "graph" of correspondence $X$, i.e.,

$$
G_{X}=\left\{(x, t) \in R^{m} \times[0, \infty) \mid x \in X(t)\right\} .
$$

Define a correspondence $F: G_{X} \rightarrow 2^{R^{\prime \prime \prime}}$ by

$$
F(x, t)=\left\{y \in R^{m} \mid(x, y) \in Y(t)\right\} .
$$

Lemma A: For any $\left(x_{0}, t_{0}\right) \in G_{X}$ with $x_{0} \in$ int $X\left(t_{0}\right)$, there exist a compact neighborhood $U$ of $x_{0}$ and two numbers $r, s \in[0, \infty)$ with $r \leqq t_{0}<s\left(r=t_{0}\right.$ only when $\left.t_{0}=0\right)$ such that $x \in$ int $X(t)$ for all $(x, t) \in U \times[r, s]$.

Lemma B: The correspondence $F$ is convex-valued and lower semi-continuous at any $\left(x_{0}, t_{0}\right) \in G_{X}$ with $x_{0} \in$ int $X\left(t_{0}\right)$.

The above two lemmas are not trivial. However we shall not prove them since their proofs are not difficult, but routine. The following is a key lemma in proving the existence of desirable feasible arcs.

Lemma C: For any $\left(x_{0}, y_{0}, t_{0}\right) \in G_{Y}$ with $x_{0} \in$ int $X\left(t_{0}\right)$, there exists a feasible arc, say $f:\left[t^{\prime}, t^{\prime \prime}\right]$ $\rightarrow R^{m}$, having the following properties:

$$
\begin{aligned}
& \left(f\left(t_{0}\right), \dot{f}\left(t_{0}\right)\right)=\left(x_{0}, y_{0}\right), \\
& t^{\prime} \leqq t_{0}<t^{\prime \prime}\left(t^{\prime}=t_{0} \text { only when } t_{0}=0\right),
\end{aligned}
$$

$$
\text { the derivative } f \text { is continuous. }
$$

ProOr: Let $\left(x_{0}, y_{0}, t_{0}\right) \in G_{Y}$ and assume that $x_{0} \in$ int $X\left(t_{0}\right)$. Then, by Lemma $\mathrm{A}$ we have a compact neighborhood $U$ of $x_{0}$ and two numbers $r, s \in[0, \infty)$ with $r \leqq t_{0}<s\left(r=t_{0}\right.$ only when $\left.t_{0}=0\right)$ such that $x \in$ int $X(t)$ for all $(x, t) \in U \times[r, s]$. Define a correspondence $H: U \times[r, s] \rightarrow 2^{R^{\prime \prime \prime}}$ 
by

$$
H(x, t)= \begin{cases}\left\{y_{0}\right\} & \text { for }(x, t)=\left(x_{0}, t_{0}\right) \\ F(x, t) & \text { for }(x, t) \neq\left(x_{0}, t_{0}\right)\end{cases}
$$

Then, by Lemma B we can easily prove that correspondence $H$ is convex-valued and lower semi-continuous. Therefore, by a continuous selection theorem of Michael [11, Theorem 3.1"'], we have a continuous function, say $h: U \times[r, s] \rightarrow R^{m}$ such that $h(x, t) \in H(x, t)$ for all $(x, t) \in U \times$ $[r . s]$. Hence, by a well-known theorem on the existence of solutions for ordinary differential equations, we have two numbers $t^{\prime}, t^{\prime \prime} \in[r, s]$ with $t^{\prime} \leqq t_{0}<t^{\prime \prime}\left(t^{\prime}=t_{0}\right.$ only when $\left.t_{0}=r\right)$ and an absolutely continuous function $f:\left[t^{\prime}, t^{\prime \prime}\right] \rightarrow R^{m}$ such that $f\left(t_{0}\right)=x_{0}$ and $\dot{f}(t)=h(f(t), t)$ for almost every $t \in\left[t^{\prime}, t^{\prime \prime}\right]$. Clearly, since $h$ is continuous, $\dot{f}$ is continuous. Also, by construction of $f$, we can easily show that $f$ is a feasible arc between time $t^{\prime}$ and time $t^{\prime \prime}$.

Let $\mathbb{B}$ be a countable base of open sets for $R^{m} \times R^{m}$. For each $B \in \mathbb{B}$, let $Y^{-1}(B)$ denote the "inverse image" of correspondence $Y$, i.e.,

$$
Y^{-1}(B)=\{t \in[0, \infty) \mid Y(t) \cap B \neq \emptyset\} .
$$

Lemma D: For any $B \in \mathbb{B}$ with $Y^{-1}(B) \neq \emptyset$, there exists a countable family of feasible arcs, say $\mathbb{F}^{B}$, satisfying the following conditions:

$$
\text { If } t \in Y^{-1}(B) \text {, then there is a feasible arc, } f:\left[t^{\prime}, t^{\prime \prime}\right] \rightarrow R^{m} \text {, }
$$

belonging to $\mathbb{F}^{B}$ such that $t^{\prime} \leqq t<t^{\prime \prime}\left(t^{\prime}=t\right.$ only when $\left.t=0\right)$,

$$
\begin{gathered}
\text { if } f:\left[t^{\prime}, t^{\prime \prime}\right] \rightarrow R^{m} \text { is a feasible arc belonging to } \mathbb{F}^{B} \text {, then } \\
(f(t), \dot{f}(t)) \in B \text { for all } t \in\left[t^{\prime}, t^{\prime \prime}\right],
\end{gathered}
$$

$$
\text { if } f \in \mathbb{F}^{B} \text {, then the derivative } \dot{f} \text { is continuous. }
$$

Proof: Let $B \in \mathbb{B}$ and assume that $Y^{-1}(B) \neq \varnothing$. Pick $s \in Y^{-1}(B)$. Then, since $B$ is open, Assumption 1 (ii) implies that there exists $\left(x_{0}, y_{0}\right) \in Y(s) \cap B$ with $x_{0} \in$ int $X(s)$. Therefore, by Lemma $\mathrm{C}$, we have a feasible arc, $f:\left[t^{\prime}, t^{\prime \prime}\right] \rightarrow R^{m}$, having the following properties:

$$
\begin{aligned}
& (f(s), \dot{f}(s))=\left(x_{0}, y_{0}\right), \\
& t^{\prime} \leqq s<t^{\prime \prime} \quad\left(t^{\prime}=s \text { only when } s=0\right),
\end{aligned}
$$

the derivative $\dot{f}$ is continuous.

Hence, since $\left(x_{0}, y_{0}\right) \in B$ and $B$ is open, we have two numbers in $\left[t^{\prime}, t^{\prime \prime}\right]$, say $t_{s}^{\prime}$ and $t_{s}^{\prime \prime}$, with $t_{s}^{\prime} \leqq s<t_{s}^{\prime \prime}\left(t_{s}^{\prime}=s\right.$ only when $\left.s=0\right)$ such that $(f(t), f(t)) \in B$ for all $t \in\left[t_{s}^{\prime}, t_{s}^{\prime \prime}\right]$. Let $f_{s}$ denote the restriction of function $f$ on $\left[t_{s}^{\prime}, t_{s}^{\prime \prime}\right]$. In this way, for each $s \in[0, \infty)$ we have a function $f_{s}:\left[t_{s}^{\prime}, t_{s}^{\prime \prime}\right]$ $\rightarrow R^{m}$.

Let $\mathbb{C}$ be a countable base of open sets for $[0, \infty)$. For each $C \in \mathbb{C}$, choose a number $s \in[0, \infty)$ and a function $f_{s}:\left[t_{s}^{\prime}, t_{s}^{\prime \prime}\right] \rightarrow R^{m}$ such that $C \subset\left[t_{s}^{\prime}, t_{s}^{\prime \prime}\right]$, if they exist. Let $\mathbb{F}^{B}$ denote the family of such functions chosen in such a way. Clearly, since $\mathbb{C}$ is a countable base, $\mathbb{F}^{B}$ is a countable family. Also, we can easily check that family $\mathbb{F}^{B}$ satisfies conditions (i), (ii), and (iii) of this lemma.

Q.E.D.

By Lemma $\mathrm{D}$, we can define a countable family of feasible arcs by

$$
\mathbb{F}=\bigcup_{B \in \mathbb{B}} \mathbb{F}^{B} .
$$


To prove that family $\mathbb{F}$ satisfies condition (i) of Assumption 2, let $t \in[0, \infty)$ and suppose that $D_{t}$ were not dense in $Y(t)$. Then, there exists $B \in \mathbb{B}$ such that $B \cap Y(t) \neq \varnothing$ and $B \cap D_{t}=\varnothing$. Since $t \in Y^{-1}(B)$, by Lemma D (i), (ii), we have a feasible arc $f:\left[t^{\prime}, t^{\prime \prime}\right] \rightarrow R^{m}$ belonging to $\mathbb{F}^{B}$ such that $t^{\prime} \leqq t<t^{\prime \prime}\left(t^{\prime}=t\right.$ only when $\left.t=0\right)$ and $(f(t), \dot{f}(t)) \in B$. Thus, $B \cap D_{t} \neq \emptyset$, which is a contradiction.

In addition, by Lemma $D$ (iii), we can easily prove that Assumption $2^{\prime}$ (ii) implies Assumption 2 (ii). Therefore, this completes the proof that Assumption $2^{\prime}$ implies Assumption 2 under Assumption 1 .

\section{REFERENCES}

[1] Aubin, J.-P., and F. H. Cl.arke: "Shadow Prices and Duality for a Class of Optimal Control Problems," SIAM Journal of Control and Optimization, 17(1979), 567-586.

[2] Benveniste, L. M., and J. A. Scheinkman: "Duality Theory for Dynamic Optimization Models of Economics: The Continuous Time Case," Discussion Paper 77-12. 1977, Department of Economics, University of Rochester, forthcoming in Journal of Economic Theory.

[3] Brock, W. A.: "On Existence of Weakly Maximal Programmes in a Multi-Sector Economy," Review of Economic Studies, 32(1970), 275-280.

[4] Brock, W. A., and A. Haurie: "On Existence of Overtaking Optimal Trajectories Over an Infinite Horizon," Mathematics of Operations Research, 1(1976), 337-346.

[5] Gal.E, D.: "On Optimal Development in a Multi-Sector Economy," Review of Economic Studies, 34(1967), 1-18.

[6] Hal.kin, H.: "Extremal Properties of Biconvex Contingent Equations," in Ordinary Differential Equations, ed. by L. Weiss. New York: Academic Press, 1972.

[7] - : "Necessary Conditions for Optimal Control Problems with Infinite Horizons," Econometrica, 42(1974), 267-272.

[8] Haurie, A.: "Optimal Control on an Infinite Time Horizon: The Turnpike Approach," Journal of Mathematical Economics, 3(1976), 81-102.

[9] McKenziE, L. W.: "Turnpike Theorems with Technology and Welfare Function Variable," in Mathematical Models in Economics, ed. by J. Kos and M. W. Kos. New York: American Elsevier, 1974, pp. 271-287.

[10] —_: "Turnpike Theory," Econometrica, 44(1976), 841-865.

[11] Michael, E.: "Continuous Selections I," Annals of Mathematics, 63(1956), 361-382.

[12] Natanson, I. P.: "Theory of Functions of a Real Variable," translated by L. F. Boron \& E. Hewitt. New York: Frederick Ungar Publishing Co., 1955.

[13] Pontryagin, L. et al.: The Mathematical Theory of Optimal Process. New York: Interscience Publishers, Inc., 1962.

[14] Rockafellar, R. T.: "Existence and Duality Theorems for Convex Problems of Bolza," Transactions of the American Mathematical Society, 159(1971), 1-40.

[15] Takfkuma, S.: "A Support Price Theorem for the Continuous Time Model of Capital Accumulation," Social Science Working Paper 243, 1979, California Institute of Technology.

[16] _ _ "Mathematical Theory of Optimal Economic Growth," Ph.D. Dissertation, submitted to the Department of Economics, University of Rochester, 1979.

[17] - "A Sensitivity Analysis on Optimal Economic Growth," Journal of Mathematical Economics, 77(1980), 193-208.

[18] von Weizsacker, C. C.: "Existence of Optimal Programs of Accumulation for an Infinite Time Horizon," Review of Economic Studies, 32(1965), 85-104.

[19] Warga, J.: "Necessary Conditions without Differentiability Assumptions in Optimal Control," Journal of Differential Equations, 18(1975), 41-62.

[20] Weitzman, M. L.: "Duality Theory for Infinite Horizon Convex Models," Management Science, 19(1973), 783-789. 